\title{
Effects of Dielectric Material Properties on Metalized Film Capacitor Performance
}

\author{
Janet Ho \\ US Army Research Laboratory \\ Adelphi, USA \\ janet.s.ho.civ@mail.mil
}

\begin{abstract}
The effects of dielectric constant and in-plane thermal conductivity of capacitor film on the maximum temperature rise per unit power dissipation or $\mathrm{K} / \mathrm{W}$ in a DC-link capacitor were studied based on finite element computations which were compared with analytical approximations. The $\mathrm{K} / \mathrm{W}$ was found to scale with the dielectric constant and the in-plane thermal conductivity according to a power-law relationship with an exponent of 0.9 and $\mathbf{- 0 . 6 5}$, respectively. While losses induced by film metallization usually dominate heat generation within a capacitor, conduction losses due to electrical conductivity higher than $10^{-13} \mathrm{~S} / \mathrm{m}$ become significant.
\end{abstract}

Keywords-metallized film capacitors; DC-link; thermal conductivity; electrical conductivity; high temperature polymers

\section{INTRODUCTION}

Alternative capacitor dielectrics to biaxially-oriented polypropylene (BOPP) with greater maximum operating temperature will facilitate more efficient design of power electronics for transportation and other systems. While many polymers have superior thermal stability to BOPP, they do not necessarily make a useful capacitor dielectric as a result of lower volumetric resistivity $\left(<10^{13} \Omega-\mathrm{m}\right)$ and higher dielectric loss $\left(>10^{-3}\right)$ at elevated temperature. In addition, thermal conductivity of the polymer plays a major role in determining temperature-rise of a capacitor. For BOPP, which is highly oriented in both machine and transverse directions resulting in a crystallinity of greater than $60 \%$, the in-plane thermal conductivity is $0.6 \mathrm{~W} / \mathrm{m}-\mathrm{K}$ [1] as compared to 0.2 to $0.4 \mathrm{~W} / \mathrm{m}$ $\mathrm{K}$ for amorphous and less oriented semicrystalline polymers. The objective of this work is to parameterize capacitor performance as a function of dielectric film material properties such as dielectric constant, thermal conductivity, and temperature-dependent electrical conductivity, for applications such as DC-link capacitors as these relationships are not well understood.

A DC-link capacitor controls ripple voltage on the bus between the inverter which converts the source voltage to a suitable DC voltage (AC to DC or DC to DC) for the power electronics. For low voltage applications $(<1 \mathrm{kV})$, DC-link capacitors are usually based on metalized film, which provides for "graceful failure". As many DC-link capacitors are used in inhomogeneous thermal environments with a heat sink, an estimate of the maximum temperature within the capacitor is

US. Government work not protected by U.S. copyright essential. This contribution analyzes the problem, with comparisons between analytical approximations and finite element computations of the maximum temperature rise per unit power dissipation or K/W to predict the "hot spot" temperature of a capacitor in an inhomogeneous thermal environment for given material properties.

Losses induced by film metallization usually dominate heat generation within a capacitor. However, conduction losses due to leakage current in the dielectric can become significant at elevated temperature as the electrical conductivity of most polymeric dielectrics increases with temperature, usually following an Arrhenius relationship with activation energy in the range of 0.5 to $1.5 \mathrm{eV}$. As a result, the utility of a polymer film capacitor at high temperature may be limited. With knowledge of maximum temperature rise per unit power dissipated $(\mathrm{K} / \mathrm{W})$, along with the thermal environment and maximum operating temperature, the allowable power dissipation which results from losses induced by metallization or leakage current can be determined.

\section{HEAT SOURCE FROM METALLIZATION}

The basic unit of a metallized film capacitor is the capacitor "winding", multiple of which can be connected in series and parallel to achieve the required voltage rating and capacitance. In the present analysis, only a single winding is modeled. For a winding of capacitance, $C$, which is composed of two single-side-metalized polymer film layers of dielectric constant, $\varepsilon$, thickness, $d$, and active width, $W$ (contributing to capacitance) wound together, the film length $L$ of each layer can be written as

$$
L=\frac{C d}{2 W \varepsilon},
$$

while equivalent series resistance, ESR, caused by film metallization is given by

$$
E S R=\frac{2 \rho W}{3 L}=\frac{4 \rho \varepsilon W^{2}}{3 d C},
$$

where $\rho$ is the surface resistivity in $\Omega /$ sq of the metallization, and the factor of $2 / 3$ results from axially-linear increase metallization current density across the active width of the metallization. 
As DC-link capacitors are often rated in terms of maximum ripple current, $I_{\mathrm{S}}$, in amps through the capacitor per micro-farad of capacitance or $\mathrm{A} / \mu \mathrm{F}$, the maximum current, $I$, through the capacitor of capacitance $C$ can be written as

$$
I=\frac{2 \varepsilon L W I_{S}}{d}
$$

Presently, the upper limit for $I_{\mathrm{S}}$ is in the range of $1 \mathrm{~A} / \mu \mathrm{F}$. Thus, the power, $P$, dissipated due to the metallization is

$$
P=I^{2} E S R=\frac{4 C W^{2} I_{S}^{2} \rho \varepsilon}{3 d} .
$$

For a given active volume $(v=2 L W d)$, the average power density, $P D_{A V}$, is

$$
P D_{A V}=\frac{P}{v}=\frac{4 W^{2} I_{S}^{2} \rho \varepsilon^{2}}{3 d^{3}} .
$$

For the finite element thermal computations to be described below, the average power density was multiplied with a quadratic function of axial position so that the power density at the axial center of the winding is $3 / 4$ the average, while the power density at the edges of the active film width is 1.5 times the average with axial integral of the function being 1 .

As shown in (5), the power density is proportional to square of the dielectric constant and the active width. Thus, optimizing the design of a capacitor winding for DC-link applications requires a delicate balance between increasing energy density, which scales linearly with dielectric constant, and controlling heat generation.

\section{FINITE ELEMENT COMPUTATIONS}

As the typical required capacitance in DC-link applications is in the range of a few hundred $\mu \mathrm{F}$ to $1 \mathrm{mF}$, the thermal model used in this contribution is based on a $1-\mathrm{mF}$ single-winding capacitor. Given the need for narrow film width, such windings will be of large diameter and would normally be heat sunk at one end, possibly by connecting the grounded end of the capacitor directly to the heat sink. In the present model, the dielectric film is $33 \mathrm{~mm}$ in active width with a thickness of 3.8 $\mu \mathrm{m}$ wound on a core of $10 \mathrm{~mm}$ in diameter, and three relative dielectric constants 2.2,3.0, and 6.0 were studied. The heat sink temperature is arbitrarily chosen as $353 \mathrm{~K}$ while two ambient temperatures 353 and $413 \mathrm{~K}$ were studied. For the latter case, the model was first equilibrated thermally with zero power density.

For convectively cooled surfaces, convective heat transfer in air with a coefficient of $12 \mathrm{~W} / \mathrm{m}^{2}-\mathrm{K}$ is used. As temperaturerise in the capacitor is sensitive to the thermal conductivity of its components, this contribution examines in-plane thermal conductivities of $0.17,0.40$ and $0.60 \mathrm{~W} / \mathrm{m}-\mathrm{K}$ for the dielectric with the through-plane value fixed at $0.16 \mathrm{~W} / \mathrm{m}-\mathrm{K}$ due to contact resistance between layers of film in an unimpregnated winding. In addition to the dielectric, the model includes a 3$\mathrm{mm}$ edge margin, 2-mm end connection with a thermal conductivity of $38 \mathrm{~W} / \mathrm{m}-\mathrm{K}$, and a 4-mm epoxy end with a thermal conductivity of $2.16 \mathrm{~W} / \mathrm{m}-\mathrm{K}$ at both ends of the winding. The surface resistivity of the film metallization is 30 $\Omega /$ sq.

\section{ANALYTICAL APPROXIMATION}

Given a winding diameter at least three times the active width and the heat sink at one end, an approximation of axial heat flow to the heat sink and no heat transfer in the radial direction is reasonable. With the assumption of an average power density throughout the active film width, an analytical approximation is available for the temperature distribution [3],

$$
T_{R}=\frac{Q W^{2}}{2 k},
$$

where $k$ is the in-plane thermal conductivity of the dielectric, $W$ is the active film width, and $Q$ the average power density. In the present thermal model, heat is lost from the top surface by convection, and the hot spot in the winding is near the top surface. To a reasonable approximation, the average power density $Q$ can be corrected for the convective heat loss from the top surface as,

$$
Q=P D_{A V}-\frac{A W^{2} h P D_{A V}}{2 k v},
$$

where $A$ is area of the top surface and $h$ the convective heat transfer coefficient from the top surface. Following from substitution of (5) and (7), (6) can be rewritten as

$$
T_{R}=\frac{I_{S}^{2} \rho W^{4} \varepsilon^{2}(2 k-W h)}{3 d^{3} k^{2}},
$$

which shows that the maximum temperature rise increases quadratically with the dielectric constant.

In a homogeneous thermal environment where the heat sink is at ambient temperature, the hot spot temperature within a capacitor can be estimated using (8). However in a thermal environment where the ambient temperature is substantially greater than that of the heat sink, the equilibrium (zero power density) temperature at the hot spot location must be known to estimate the maximum winding temperature. Assuming convective heat transfer at top end of the winding and ignoring radial heat transfer, the thermal resistance, $R_{T}$, from top to bottom surface capacitor (axial direction) can be written as

$$
R_{T}=\frac{1}{2 L d}\left[\frac{W}{k}+2\left(\frac{W_{E M}}{k_{E M}}+\frac{W_{E C}}{k_{E C}}+\frac{W_{E P}}{k_{E P}}\right)\right],
$$

where $E M$ stands for edge margin, $E C$ end connection, $E P$ epoxy case. The power entering the top surface, $P_{T O P}$, as a result of convective heat transfer is given by

$$
P_{T O P}=\frac{\Delta T_{i}}{R_{T}}=2 L d\left(T_{A}-T_{S}-\Delta T_{i}\right) h,
$$

where $T_{A}$ is the ambient temperature, $T_{S}$ the heat sink temperature, $\Delta T_{i}$ the temperature difference across the 
capacitor in the axial direction at equilibrium with zero power density. Eq. (10) can be rewritten as

$$
2 L d\left(T_{A}-T_{S}-\Delta T_{i}\right) h R_{T}-\Delta T_{i}=0
$$

from which, $\Delta T_{i}$ can be determined as follows

$$
\Delta T_{i}=\frac{h\left(T_{A}-T_{S}\right)\left(W+2 W_{E M}+k_{e f f} k\right)}{k\left(1+h k_{e f f}\right)+h\left(W+2 W_{E M}\right)},
$$

where $k_{\text {eff }}=2\left(\left(W_{E C} / k_{E C}\right)+\left(W_{E P} / k_{E P}\right)\right)$.

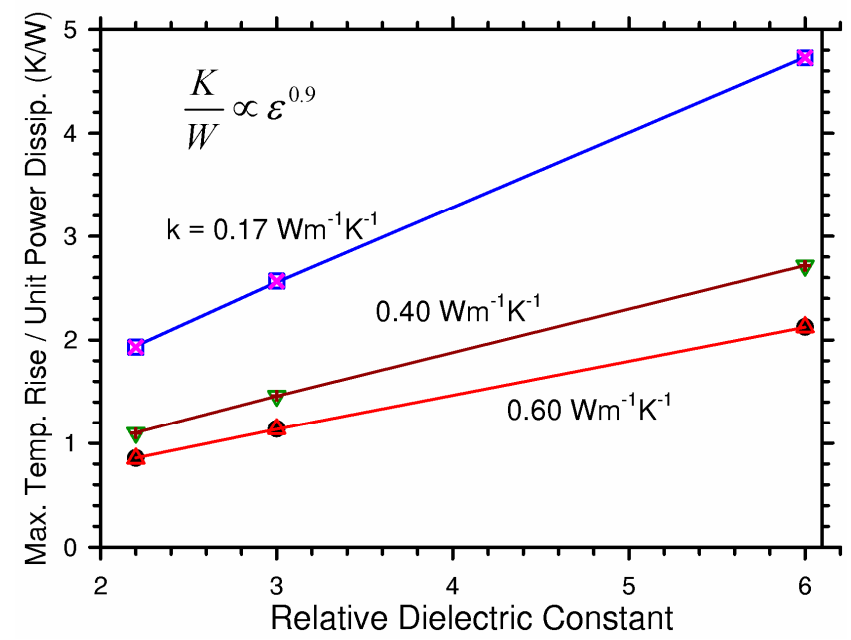

Fig. 1. Maximum temperature rise per unit power dissipation computed from the finite element model of a $1-\mathrm{mF}$ single-winding capacitor heat sunk at one end at $353 \mathrm{~K}$ in an ambient of $353 \mathrm{~K}$ or $413 \mathrm{~K}$ (symbols overlapped in the plot) with a ripple current of $1 \mathrm{~A} / \mu \mathrm{F}$ for various dielectric constants and inplane thermal conductivities of the capacitor film. Solid line: $y=a * x^{0.9}$

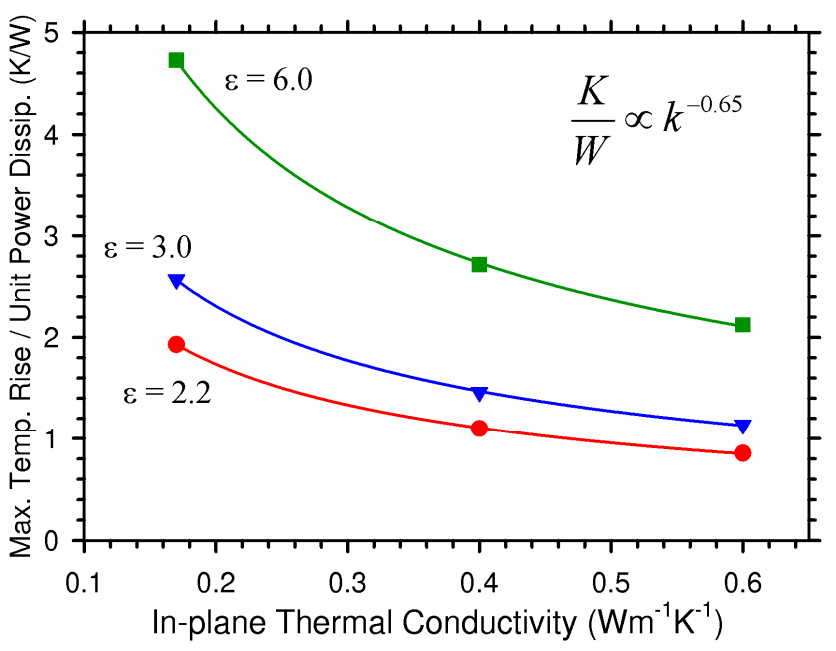

Fig. 2. Maximum temperature rise per unit power dissipation computed from the finite element model of a $1-\mathrm{mF}$ single-winding capacitor heat sunk at one end at $353 \mathrm{~K}$ in an ambient of $413 \mathrm{~K}$ with a ripple current of $1 \mathrm{~A} / \mu \mathrm{F}$ for various in-plane thermal conductivities and dielectric constants of the capacitor film. Solid line: $y=a * x^{-0.65}$

\section{CAPACITOR THERMAL RATING}

DC-link capacitors often have a specified " $\mathrm{K} / \mathrm{W}$ ", which is the maximum temperature rise per Watt dissipated in the capacitor. As the DC-link capacitors are presently made from BOPP which has a relative dielectric constant of 2.2 and an inplane thermal conductivity of $0.6 \mathrm{~W} / \mathrm{m}-\mathrm{K}$, the objective of this contribution is to understand the effect of dielectric constant and thermal conductivity of the capacitor dielectric on the thermal rating. Figure 1 shows this parameter computed from a finite element model of a 1-mF, single-winding capacitor heat sunk from one end at $353 \mathrm{~K}$ in two different ambient temperatures 353 and $413 \mathrm{~K}$, with a ripple current rating of 1 $\mathrm{A} / \mu \mathrm{F}$ for film relative dielectric constants of $2.2,3.0$, and 6.0 and in-plane thermal conductivities of $0.17,0.40$, and 0.60 $\mathrm{W} / \mathrm{m}-\mathrm{K}$. Results from the thermal computations suggest that for such a capacitor with heat sunk at one end, the ambient temperature does not seem to affect the $\mathrm{K} / \mathrm{W}$ value as indicated by the overlapping of the data shown in Figure 1 for a given dielectric constant and thermal conductivity. A curve fit to the data gives an approximately linear relationship between $\mathrm{K} / \mathrm{W}$ and the dielectric constant. The $\mathrm{K} / \mathrm{W}$ increases with dielectric constant as a result of the decreasing winding cross section $(2 L d)$ for axial heat flow with increasing dielectric constant as the length $L$ is inversely proportional to the dielectric constant. Also shown in Figure 1 is the $\mathrm{K} / \mathrm{W}$ computed at various inplane thermal conductivity for a given dielectric constant. The $\mathrm{K} / \mathrm{W}$ decreases with increasing thermal conductivity and exhibits a power-law relationship with an exponent of -0.65 as illustrated in Figure 2.

While finite element computations can provide more reliable information for complicated systems if performed correctly, analytical approximations may be used to gain some "engineering insight" for simple systems. Figure 3 compares the $\mathrm{K} / \mathrm{W}$ value using the maximum temperature rise computed by the approximation derived in (8) which assumes no heat transfer in the radial direction. For an in-plane film thermal conductivity of $0.6 \mathrm{~W} / \mathrm{m}-\mathrm{K}$, the approximation agrees well with the finite element computations while for $0.4 \mathrm{~W} / \mathrm{m}-\mathrm{K}$ in-plane film thermal conductivity, the approximation underestimates slightly. For an in-plane thermal conductivity of $0.17 \mathrm{~W} / \mathrm{m}-\mathrm{K}$ (not shown), the approximation is not applicable, as the radial heat transfer becomes significant relative to the axial heat transfer. Given that many DC-link capacitors are used in inhomogeneous thermal environments with a heat sink, the equilibrium (zero power density) temperature at the hot spot location must be known in order to estimate the maximum winding temperature. Table 1 compares the equilibrium temperature at zero power density computed by finite element computations and based on the approximation in (12) for the three in-plane thermal conductivities. For an in-plane thermal conductivity of $0.17 \mathrm{~W} / \mathrm{m}-\mathrm{K}$, the approximation shows poor agreement with the finite element computation while for inplane thermal conductivities of 0.4 and $0.6 \mathrm{~W} / \mathrm{m}-\mathrm{K}$, the agreement is good. 


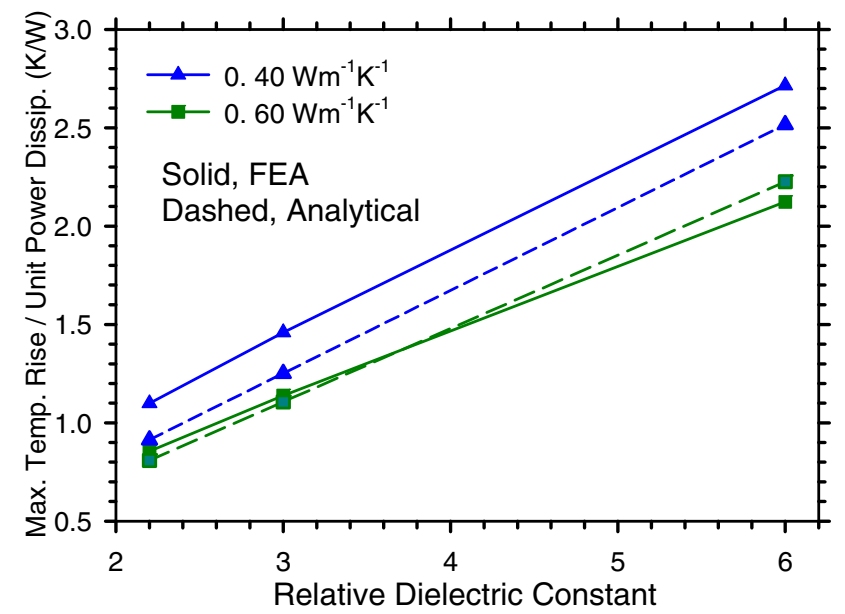

Fig. 3. Comparison between the analytical approximation given in (8) and finite element computation of the maximum temperature rise of a $1-\mathrm{mF}$ single-winding capacitor heat sunk at one end at $353 \mathrm{~K}$ in an ambient of 413 $\mathrm{K}$ with a ripple current of $1 \mathrm{~A} / \mu \mathrm{F}$ for various dielectric constants and in-plane thermal conductivities of the capacitor film. Solid line: guide to the eye.

TABLE I. EQUILIBRIUM TEMPERATURE AT ZERO POWER DENSITY OF THE MODEL CAPACITOR HEAT SUNK AT ONE END AT $353 \mathrm{~K}$ IN AN AMBIENT OF 413 K FOR VARIOUS IN-PLANE THERMAL CONDUCTIVITIES.

\begin{tabular}{|c|c|c|}
\hline \multirow{2}{*}{$\begin{array}{c}\text { Thermal } \\
\text { Conductivity, k, } \\
\left(\mathbf{W m}^{-1} \mathbf{K}^{-1}\right)\end{array}$} & \multicolumn{2}{|c|}{$\begin{array}{c}\text { Equilibrium Temperature at Zero } \\
\text { Power Density, (K) }\end{array}$} \\
\cline { 2 - 3 } & $\begin{array}{c}\text { Finite Element } \\
\text { Computations }\end{array}$ & $\begin{array}{c}\text { Analytical } \\
\text { Approximation }\end{array}$ \\
\hline 0.17 & 385 & 397 \\
\hline 0.40 & 382 & 386 \\
\hline 0.60 & 379 & 380 \\
\hline
\end{tabular}

\section{LOSSES DUE TO LEAKAGE CURRENT}

While losses induced by film metallization usually dominate heat generation within a capacitor, conduction losses caused by leakage current in the dielectric can become significant at elevated temperature as the electrical conductivity of most polymeric dielectrics increases with temperature, usually following an Arrhenius relationship. Such relationship for BOPP with activation energy of $0.75 \mathrm{eV}$ was previously determined [3]. With the maximum temperature rise per unit power dissipation determined above, the maximum temperature rise caused by conduction losses assuming the same Arrhenius behavior was computed for the various dielectric constants and in-plane thermal conductivities as shown in Figure 4. For an electrical conductivity below $10^{-13}$ $\mathrm{S} / \mathrm{m}$, the resulting maximum temperature rise is in the range of 10 to $20 \mathrm{~K}$ while for $10^{-12} \mathrm{~S} / \mathrm{m}$, the maximum temperature rise becomes inhibiting.

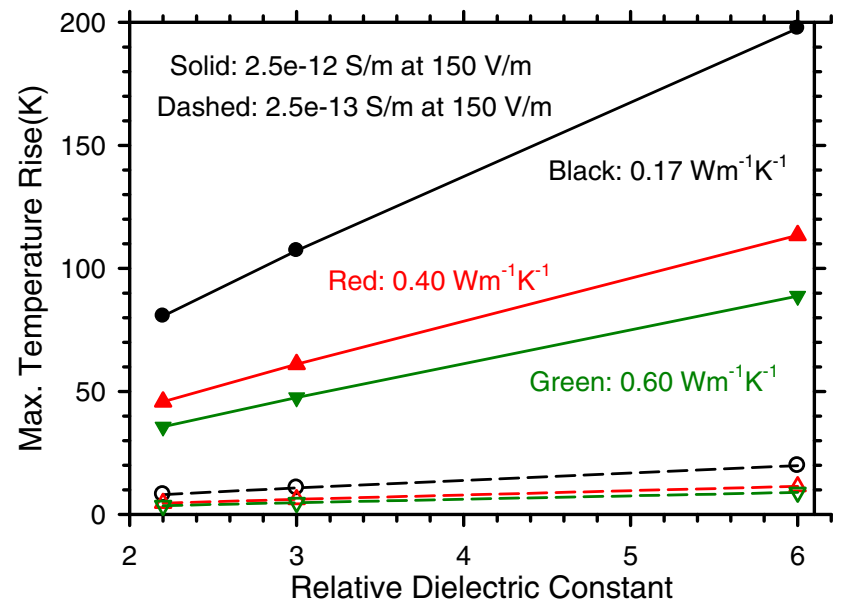

Fig. 4. Maximum temperature rise due to conduction losses caused by electrical conductivities of $2.5 \mathrm{e}-12 \mathrm{~S} / \mathrm{m}$ (solid line) and $2.5 \mathrm{e}-13 \mathrm{~S} / \mathrm{m}$ (dashed line) based on the $\mathrm{K} / \mathrm{W}$ computed from the finite element model.

\section{CONCLUSION}

This contribution examines the effects of dielectric constant and in-plane thermal conductivity of capacitor film on the maximum temperature rise per unit power dissipation or $\mathrm{K} / \mathrm{W}$ in a DC-link capacitor based on finite element computations of a 1-mF single-winding capacitor heat sunk at $353 \mathrm{~K}$ at one end in an ambient of $353 \mathrm{~K}$ and $413 \mathrm{~K}$. The results were compared with analytical approximations derived assuming heat flows only in the axial direction with no heat transfer radially. The $\mathrm{K} / \mathrm{W}$ was found to scale with the dielectric constant and the inplane thermal conductivity according to a power-law relationship with an exponent of 0.9 and -0.65 , respectively. While losses induced by film metallization usually dominate heat generation within a capacitor, conduction losses due to electrical conductivity higher than $10^{-13} \mathrm{~S} / \mathrm{m}$ become significant.

\section{REFERENCES}

[1] S. Qin, J. Ho, M. Rabuffi, G. Borelli, T.R. Jow, "Implications of the anisotropic thermal conductivity of capacitor windings," IEEE Electrical Insulation Magazine, vol. 27, No. 1, pp. 7-13, 2011.

[2] J.S. Carslaw and J.C. Jaeger, Conduction of Heat in Solids, 1st ed., Oxford: Clarendon, 1948, pp. 108.

[3] J. Ho and T.R. Jow, "High field conduction in biaxially oriented polypropylene at elevated temperature," IEEE Trans. Diele. Electr. Insul., vol. 19, No. 3, pp. 990-995, 2012 